\title{
Drug and Chemical Glucosidation by Control Supersomes and Membranes from Spodoptera frugiperda (Sf) 9 Cells: Implications for the Apparent Glucuronidation of Xenobiotics by UDP-glucuronosyltransferase $1 \mathrm{A5}^{\mathrm{S}}$
}

\author{
Nuy Chau, Leyla Kaya, Benjamin C. Lewis, Peter I. Mackenzie, and John O. Miners \\ Department of Clinical Pharmacology (N.C., L.K., B.C.L., P.I.M., J.O.M.) and Flinders Centre for Innovation in Cancer (B.C.L., P.I.M., \\ J.O.M.), Flinders University College of Medicine and Public Health, Adelaide, Australia
}

Received October 21, 2018; accepted November 29, 2018

\begin{abstract}
Accumulating evidence indicates that several human UDPglucuronosyltransferase (UGT) enzymes catalyze both glucuronidation and glucosidation reactions. Baculovirus-infected insect cells [Trichoplusia ni and Spodoptera frugiperda (Sf9)] are used widely for the expression of recombinant human UGT enzymes. Following the observation that control Supersomes (c-SUP) express a native enzyme capable of glucosidating morphine, we characterized the glucosidation of a series of aglycones with a hydroxyl (aliphatic or phenolic), carboxylic acid, or amine functional group by c-SUP and membranes from uninfected Sf9 cells. Although both enzyme sources glucosidated the phenolic substrates investigated, albeit with differing activities, differences were observed in the selectivities of the native UDP-glucosyltransferases toward aliphatic alcohols, carboxylic acids, and amines. For example, zidovudine was solely glucosidated by c-SUP. By contrast, c-SUP lacked activity
\end{abstract}

toward the amines lamotrigine and trifluoperazine and did not form the acyl glucoside of mycophenolic acid, reactions all catalyzed by uninfected Sf9 membranes. Glucosidation intrinsic clearances were high for several substrates, notably 1-hydroxypyrene ( 1400-1900 $\mu \mathrm{l} / \mathrm{min} \cdot \mathrm{mg})$. The results underscore the importance of including control cell membranes in the investigation of drug and chemical glucosidation by UGT enzymes expressed in T. ni (High-Five) and Sf9 cells. In a coincident study, we observed that UGT1A5 expressed in Sf9, human embryonic kidney 293T, and COS7 cells lacked glucuronidation activity toward prototypic phenolic substrates. However, Sf9 cells expressing UGT1A5 glucosidated 1-hydroxypyrene with UDP-glucuronic acid as the cofactor, presumably due to the presence of UDP-glucose as an impurity. Artifactual glucosidation may explain, at least in part, a previous report of phenolic glucuronidation by UGT1A5.

\section{Introduction}

Enzymes of the UDP-glucuronosyltransferase (UGT) family play a pivotal role in the clearance and detoxification of a structurally diverse range of substrates that include drugs, nondrug xenobiotics, and endogenous compounds. The 19 human UGT proteins classified in subfamilies $1 \mathrm{~A}, 2 \mathrm{~A}$, and 2B primarily catalyze the transfer of glucuronic acid from the cofactor UDP-glucuronic acid (UDP-GlcUA) to a typically lipophilic substrate (or aglycone) bearing a nucleophilic "acceptor" functional group to form a glucuronide conjugate that is excreted in urine and/or bile (Mackenzie et al., 2005; Miners et al., 2010). By contrast, UGT 3A1 and 3A2 use UDP-sugars other than UDP-GlcUA [e.g., UDPglucose (UDP-Glc), UDP-xylose, and UDP- $N$-acetylglucosamine] as the cofactor (Mackenzie et al., 2008, 2011). Although glucuronidation is the major metabolic pathway mediated by UGT1A, 2A, and $2 \mathrm{~B}$ enzymes, $1 \mathrm{~A}$ and $2 \mathrm{~B}$ subfamily enzymes may additionally use sugar

https://doi.org/10.1124/dmd.118.084947

SThis article has supplemental material available at dmd.aspetjournals.org. donors other than UDP-GlcUA, especially UDP-Glc. In particular, UGT2B7 catalyzes both the glucuronidation and glucosidation of a number of substrates, including morphine (MOR), forming phenolic-, acyl-, and $N$-glucosides (Mackenzie et al., 2003; Tang et al., 2003; Toide et al., 2004; Buchheit et al., 2011; Chau et al., 2014). At least with MOR glycosidation by UGT2B7, glucuronidation predominates over glucosidation because the binding affinity of UDP-GlcUA is higher than that of UDP-Glc (Chau et al., 2014). Substrates of other UGT enzymes (e.g., 1A1, 1A9, and 2B10) have also been reported to form glycoside conjugates other than glucuronides (Fevery et al., 1977; Senafi et al., 1994; Lu et al., 2018; Chau and Miners, unpublished data). Despite the likelihood that UGT-catalyzed glucuronidation and glucosidation of xenobiotics may occur as complementary metabolic pathways, glucosidation has received little attention (Tang, 1990; Meech et al., 2012).

It is well established that the individual human UGT enzymes exhibit distinct, but frequently overlapping, substrate and inhibitor selectivities (Miners et al., 2004, 2010). In this regard, the availability of cDNAexpressed UGT proteins has been pivotal in the characterization of UGT function. Recombinant UGTs have been expressed in numerous

ABBREVIATIONS: 1-OHP, 1-hydroxypyrene; 1-NAP, 1-naphthol; 21-OHPr, 21-hydroxyprogesterone; 4-MU, 4-methylumbelliferone; 4-NP, 4nitrophenol; AZT, zidovudine; BZC, benzocaine; COD, codeine; c-SUP, control Supersomes; HEK293T, human embryonic kidney 293T; HPLC, high-performance liquid chromatography; LC-MS, liquid chromatography-mass spectrometry; LTG, lamotrigine; MOR, morphine; MPA, mycophenolic acid; MS, mass spectrometry; PE, phenethyl alcohol; Sf9, clonal isolate from Spodoptera frugiperda IPLB-Sf21-AE cells; Sf9 membranes, membranes prepared from uninfected Sf9 cells; S-NAP, S-naproxen; TFP, trifluoperazine; UDP-Glc, UDP-glucose; UDP-GlcUA, UDPglucuronic acid; UGT, UDP-glucuronosyltransferase; UPLC, Ultra Performance Liquid Chromatography. 
mammalian and nonmammalian cell lines (Radominska-Pandya et al., 2005). Examples include COS (African green monkey kidney fibroblasts), human embryonic kidney 293T cell line (HEK293T), V79 (Chinese hamster lung fibroblasts), yeast (Pichia pastoris and Saccharomyces cerevisiae), and baculovirus-infected insect cells [Spodoptera frugiperda (Sf9) and Trichoplusia ni] (e.g., Fournel-Gigleux et al., 1991; Jin et al., 1997; Nguyen and Tukey, 1997; Ouzzine et al., 1999; Uchaipichat et al., 2004; Zhang et al., 2012). Of these, the use of commercially available UGT-expressing Supersomes, prepared from baculovirus-infected $T$. $n i$ cells, has become widespread in academia and industry. However, in a recent study of the comparative 3- glucuronidation and glucosidation of MOR by UGT2B7, we observed that Supersomes expressing UGT2B4, 2B15, and 2B17 protein as well as control Supersomes [c-SUP; insect cell "control" microsomes prepared from $T$. $n i$ (High-Five) cells infected with wild-type baculovirus] all exhibited significant and comparable MOR 3-glucosidation activities (Chau et al., 2014).

Since these data indicate that Supersomes express a "native" enzyme capable of MOR 3-glucosidation, we characterized the glucosidation of a series of aglycones with a phenolic [1-hydroxypyrene (1-OHP), 4-methylumbelliferone (4-MU), MOR, mycophenolic acid (MPA), 1-naphthol (1-NAP), and 4-nitrophenol (4-NP)], aliphatic alcohol [codeine (COD), 21-hydroxyprogesterone (21-OHPr), phenethyl alcohol (PE), and zidovudine (AZT)], acyl [MPA, S-naproxen (S-NAP)], or amine [benzocaine (BZC), lamotrigine (LTG), and trifluoperazine (TFP)] acceptor functional group (see Supplemental Fig. 1 for structures and sites of conjugation) by c-SUP. The glucosidation of these aglycones was additionally characterized using the enriched membrane fraction from uninfected Sf9 cells (subsequently referred to as "Sf9 membranes") since baculovirus-infected Sf9 cells are also used for UGT expression (e.g., Zhang et al., 2012) and are available commercially as Baculosomes.

Coincident with these studies, we conducted an investigation of UGT1A5 structure function. UGT1A5 expressed in baculovirusinfected Sf9 cells has been reported to glucuronidate a number of phenolic substrates, including 1-OHP and 4-MU (Finel et al., 2005). However, we found that UGT1A5 lacked glucuronidation activity when expressed in COS7, HEK293T, and baculovirus-infected Sf9 cells. The glucosidation activity studies reported here indicate that 1-OHP, 4-MU, and most other aglycones investigated are glucosidated by c-SUP and/or Sf9 cell membranes. It is possible that artifactual glucosidation by Sf9 membranes may contribute, at least in part, to the differing UGT1A5 glucuronidation data reported between laboratories.

\section{Materials and Methods}

\section{Materials}

AZT, AZT $\beta$-D-glucuronide, COD, gentamicin, 21-OHPr, 1-OHP, kanamycin, 4-MU, 4-MU $\beta$-D-glucoside, 1-NAP, 1-NAP $\beta$-D-glucuronide, S-NAP, 4-NP, 1-octanesulfonic acid sodium salt, tetracycline, TFP, triethylamine, UDP-Glc (disodium salt), and UDP-GlcUA (trisodium salt) were purchased from SigmaAldrich (Sydney, NSW, Australia); BZC, COD $\beta$-D-glucuronide, 1-OHP $\beta$-Dglucuronide, MPA, MPA acyl $\beta$-D-glucoside, MPA phenolic $\beta$-D-glucoside, PE, and PE $\beta$-D-glucoside were from Toronto Research Chemicals (Toronto, ON, Canada); 4-NP $\beta$-D-glucoside was from Molekula Limited (Dorset, UK); BZC $N$ glucoside was from Dalton Pharma Services (Toronto, ON, Canada); and MOR hydrochloride was from GlaxoSmithKline (Melbourne, VIC, Australia). LTG and LTG N2-glucuronide were a gift from the Wellcome Foundation Ltd. (London, UK). MOR 3- $\beta$-D-glucoside was synthesized in house as described by Chau et al. (2014). Microsomes from High-Five cells infected with "control" (wild-type) baculovirus (c-SUP) were purchased from Corning Gentest (BD Biosciences, North Ryde, NSW, Australia); uninfected Sf9 cells, penicillin-streptomycin solution (100 U/ml and $100 \mu \mathrm{g} / \mathrm{ml})$, Cellfectin reagent, and DH10Bac
Escherichia coli cells were from Invitrogen (Carlsbad, CA); COS7 and HEK293 cells were from American Type Culture Collection (Manassas, VA); and Hyclone SFX-Insect Cell Culture medium and heat-inactivated fetal bovine serum were from Thermo Fisher Scientific (Waltham, MA). Solvents and other reagents were of analytical reagent grade.

\section{Methods}

Glucosidation Assay. Incubations, in a total volume of $200 \mu$ l, contained phosphate buffer $(0.1 \mathrm{M}$, pH 7.4 or $\mathrm{pH} 6.8$ for carboxylic acid-containing substrates), $\mathrm{MgCl}_{2}(4 \mathrm{mM})$, uninfected $\mathrm{Sf} 9$ cell membranes $(1 \mathrm{mg} / \mathrm{ml})$, substrate, and UDP-Glc $(5 \mathrm{mM})$. After a 5 -minute preincubation at $37^{\circ} \mathrm{C}$ in a shaking water bath, reactions were initiated by the addition of UDP-Glc and performed for 2 hours. Reactions were terminated by the addition of perchloric acid [70\% (v/v); $2 \mu \mathrm{l}$ ], ascorbic acid in methanol [2\% (w/v); $200 \mu \mathrm{l}$ ], or acetic acid in methanol [4\% (v/v); $200 \mu \mathrm{l}]$, depending on the substrate (Supplemental Table 1), and were cooled on ice for 10 minutes. Samples were centrifuged ( $5000 \mathrm{~g}$ for 10 minutes), and a 5- to $40-\mu 1$ aliquot of the supernatant fraction was analyzed by highperformance liquid chromatography (HPLC). Rates of glucoside formation were measured at four different substrate concentrations (see Results). Experiments utilizing c-SUP as the enzyme source were as described for Sf9 membranes, except the incubation volume was $100 \mu$ l. Incubations were performed at least in duplicate ( $<5 \%$ variance between replicates). Incubations for mass spectrometry (MS) analysis followed the aforementioned protocols, except reactions were terminated by the addition of 2 volumes of MS-grade $4 \%$ acetic acid in methanol or $2 \%$ ascorbic acid in methanol (BZC glucosidation assay).

Incubation conditions for studies characterizing glucosidation kinetic parameters for 1-OHP, MPA (phenolic and acyl), MOR, and 4-MU with both c-SUP and uninfected Sf9 membranes as the enzyme source were as described earlier, with the following changes to protein concentrations and incubation times: 1-OHP $(0.01 \mathrm{mg} / \mathrm{ml}, 15$ minutes$)$, MOR ( $1 \mathrm{mg} / \mathrm{ml}, 60$ minutes $),$ MPA $(0.1 \mathrm{mg} / \mathrm{ml}$, 15 minutes), and 4-MU ( $0.1 \mathrm{mg} / \mathrm{ml}, 30$ minutes). Kinetic studies included 11 or 12 substrate concentrations that spanned the $\mathrm{K}_{\mathrm{m}}\left(\right.$ or $\left.\mathrm{S}_{50}\right)$.

Quantification of Glucoside Conjugate Formation by HPLC. Glucoside conjugates were measured by reversed-phase HPLC using an Agilent 1100 series instrument (Agilent Technologies, Sydney, Australia) comprising an autoinjector, a quaternary solvent delivery system, and a UV detector (1200 series). Analytes were separated using varying chromatographic conditions, depending on the aglycone. Columns, mobile phases, absorbance wavelengths, precipitating agent, retention times of glucosides, detection method and wavelength, and injection volume are given in Supplemental Table 1. Glucoside formation was quantified by comparison of peak areas to those of a standard curve; authentic glucoside conjugates were available for BZC, 21-OHPr, MPA, MOR, 4-MU, and 4-NP. Where the glucoside was unavailable, either the corresponding glucuronide (AZT, COD, LTG, 1-NAP, and 1-OHP) or aglycone (S-NAP and TFP) was used for standard curve generation. The identity of the glucoside conjugates was confirmed by cochromatography with the authentic standard (where available) and from the $\mathrm{m} / \mathrm{z}$ ratios and fragmentation patterns generated by liquid chromatography-mass spectrometry (LC-MS) (described later). Calibration curves included five concentrations, the ranges of which are given in Supplemental Table 1.

Confirmation of Glucoside Formation by Ultraperformance Liquid Chromatography-MS. Glucoside conjugates were separated and detected using a Waters ACQUITY Ultra Performance Liquid Chromatography (UPLC) system coupled to a Waters Micromass Q-TOF Premier mass spectrometer (Waters Corporation Micromass UK Ltd., Manchester, UK). Analytes were separated on an ACQUITY UPLC HSST3 column $(1.8-\mu \mathrm{m}$ particle size, $2.1 \times 100 \mathrm{~mm}$; Waters Corporation, Milford, MA). The mobile phase, delivered at a flow rate of $0.25 \mathrm{ml} / \mathrm{min}$, consisted of two solutions (phase A, 100\% MS-grade acetonitrile; phase $\mathrm{B}, 5 \%$ acetonitrile in water) mixed according to a gradient timetable. Initial conditions were $5 \%$ phase A-95\% phase B held for 3 minutes followed by a linear gradient over 7 minutes to $60 \%$ phase $\mathrm{A}-40 \%$ phase $\mathrm{B}$, which was held constant for 0.5 minutes. The total run time, including reconditioning of the column to initial conditions, was 12.5 minutes. The MS was operated in positive ion mode with electrospray ionization. Time-of-flight data were acquired in selected ion mode, where the first resolving quadrupole acquired mass data from $\mathrm{m} / \mathrm{z} 100$ to 1000. Collision cell energy alternated between $2 \mathrm{eV}$ and a high-energy ramp (3$15 \mathrm{eV}$ ). The cone and desolvation gases were set to flow rates of 50 and $550 \mathrm{l} / \mathrm{h}$, respectively; desolvation and source temperatures were $250^{\circ} \mathrm{C}$ and $90^{\circ} \mathrm{C}$, 
respectively; and capillary and cone voltages were 1800 and $25 \mathrm{~V}$, respectively. MS data were collected as total ion chromatograms, with selected ion (pseudo multiple reaction monitoring) data extracted at the $\left[\mathrm{M}+\mathrm{H}^{+}\right]$for each analyte of interest using Waters QuanLynx software (Waters Corporation).

Construction of Recombinant Baculovirus. The preparation of the human UGT1A5 cDNA (NM_019078) from epithelial colorectal adenocarcinoma (Caco-2) cells has been described previously by Finel et al. (2005). For expression in Sf9 cells, the cDNA was subcloned into the pFastBac-HT vector (Invitrogen) using XhoI and HindIII restriction sites, and the engineered pFB-UGT1A5-His sequence was confirmed on both strands (ABI 3130-XL DNA sequencer; Applied Biosystems, Vic Australia). Generation of recombinant Bacmid DNA was achieved by transposition of the UGT1A5 cDNA from pFastBac-HT (1 ng) to the viral genome of DH10Bac chemically competent $E$. coli cells $(50 \mu \mathrm{l})$. Recombinant Bacmid DNA was amplified (100-ml culture) and purified (Plasmid Midi Kit; Qiagen, Hilden, Germany). Polymerase chain reaction analysis of the recombinant Bacmid DNA was performed to identify the presence of UGT1A5 in the $A c \mathrm{MNPV}$ viral genome using the $\mathrm{pUC} / \mathrm{M} 13$ forward and reverse primers.

Expression of UGT1A5 in Sf9 Cells and Separation of Sf9 Membranes. Sf9 cells adapted to suspension culture were grown in SFX-Insect medium supplemented with heat-inactivated fetal bovine serum $(5 \%)$ and penicillinstreptomycin (1000 U and $1 \mathrm{mg})$. Cells were seeded $\left(5 \times 10^{5} \mathrm{cell} / \mathrm{ml}\right)$ and cultured in glass impeller spinner flasks (Bellco Glass, Inc., Vineland, NJ) at $28^{\circ} \mathrm{C}$ and $120 \mathrm{rpm}$ (50\% spinner volume) in exponential growth phase with a cell density between $1 \times 10^{6}$ and $2.5 \times 10^{6}$ cells $/ \mathrm{ml}$ at greater than $95 \%$ viability. Infection optimization for UGT1A5 expression was undertaken in monolayer cultures of Sf9 cells using the Cellfectin method described by the manufacturer (Invitrogen). Large-scale expression of UGT1A5 was performed in 1-1 shaker flasks with Sf9 cells in midlogarithmic growth at a seeding density of $1 \times 10^{6}$ cell $/ \mathrm{ml}$. Cells were infected with AcMNPV-UGT1A5 at multiplicity of infection $10\left(150 \mu \mathrm{l} ; 2 \times 10^{8}\right.$ pfu/ml) and harvested 48 hours postinfection by centrifugation at $850 \mathrm{~g}$ for 10 minutes.

The enriched membrane fraction from both Sf9 cells infected with UGT1A5containing Bacmid (i.e., expressing UGT1A5) and uninfected Sf9 cells was isolated by sonication and ultracentrifugation. Pelleted cells were resuspended in cold deionized water $(0.33 \mathrm{~g}$ pellet $/ \mathrm{ml})$; homogenized with 15 strokes using a Potter-Elvehjem homogenizer; sonicated by eight 1-second "bursts," each separated by 1-minute cooling on ice, using a Vibra Cell VCX 130 Ultrasonics Processor (Sonics and Materials, Newton, CT); and then centrifuged at 10,000g for 10 minutes at $4^{\circ} \mathrm{C}$. The supernatant fraction was decanted and centrifuged at $105,000 \mathrm{~g}$ at $4^{\circ} \mathrm{C}$ for 75 minutes. The pellet, which comprised the enriched membrane fraction, was resuspended in phosphate buffer $(0.1 \mathrm{M}, \mathrm{pH} 7.4)$ and stored at $-80^{\circ} \mathrm{C}$ until use.

The UGT1A5 cDNA, cloned in the pEF-IRES-puro 6 vector, was stably expressed in HEK293T and COS7 cells using the procedure described by Uchaipichat et al. (2004), and cell lysates were used for immunoblotting and assessment of enzyme activity.

Immunoblotting. Cell lysates from transfected HEK293T and COS7 cells and Sf9 membranes expressing UGT1A5 protein $(20-100 \mu \mathrm{g})$ were separated by $10 \%$ SDS-polyacrylamide gel electrophoresis and rectilinearly transferred to nitrocellulose $(0.45 \mu \mathrm{m}$; Bio-Rad Laboratories, Hercules, CA). Immunodetection of UGT1A5 protein was performed using the WB-Human UGT1A Western Blotting Kit (BD Gentest, Woburn, MA). Nitrocellulose membranes were incubated with rabbit anti-UGT1A subfamily IgG as the primary antibody (1:1500 dilution) followed by horseradish peroxidase-conjugated goat anti-rabbit IgG (Thermo Scientific, Rockland, IL) as the secondary antibody (1:2000 dilution). Additionally, Sf9-expressed UGT1A5 was probed with His-tag-recognizing primary polyclonal antisera (rabbit anti-human His-tagged UGT2B7) developed in this laboratory (Kerdpin et al., 2009). This antibody was raised to residues 55-165 of UGT2B7 and expressed in E. coli with a 6-histidine tag at the C terminus, and hence recognized His-tagged proteins. The primary antiserum (1:1500 dilution) was detected using horseradish peroxidase-conjugated goat anti-rabbit $\operatorname{IgG}(1$ : 2000 dilution). Immunoreactivity was detected by chemiluminescence (Roche Diagnostics GmbH, Mannheim, Germany). Blots were visualized with a Fujifilm LAS-4000 imaging system (Fujifilm Life Sciences, NSW, Australia), and band intensities were measured using Multi Gauge software (Fujifilm Life Sciences). Relative UGT1A5 protein levels represent the mean of triplicate measurements. Western blot analysis and activity assays were performed using the same batch of cell lysate.
Data Analysis. Activity and kinetic data from experiments using uninfected $\mathrm{Sf} 9$ membranes and c-SUP represent the mean of duplicate measurements unless otherwise indicated. For generation of kinetic constants, the Michaelis-Menten, Hill, and substrate inhibition equations were fit to untransformed experimental data using Enzfitter (Biosoft, Cambridge, UK) to generate kinetic parameters. Goodness of fit was assessed from the coefficient of determination $\left(r^{2}\right)$, F-statistic, 95\% confidence intervals, and S.E. of the fit. Duplicate data were pooled for model fitting. Kinetic data are shown as Eadie-Hofstee plots (velocity vs. velocity/ [substrate]), and kinetic constants are reported as the parameter \pm S.E. of the parameter fit.

Michaelis-Menten equation.

$$
v=\frac{\mathrm{V}_{\max } \times[S]}{\mathrm{K}_{\mathrm{m}}+[S]}
$$

where $v$ is the rate of metabolite formation, $\mathrm{V}_{\max }$ is the maximum velocity (as $\mathrm{pmol} / \mathrm{min} \cdot \mathrm{mg}$ microsomal or cell lysate protein), $[S]$ is the substrate concentration, and $\mathrm{K}_{\mathrm{m}}$ is the Michaelis constant (substrate concentration at $0.5 V_{\max }$ ).

Hill equation.

$$
v=\frac{\mathrm{V}_{\max } \times[S]^{n}}{\mathrm{~S}_{50}+[S]^{n}}
$$

where $\mathrm{S}_{50}$ is the Hill constant (substrate concentration at $0.5 \mathrm{~V}_{\max }$ ), and $n$ is the Hill coefficient $(n<1=$ negative cooperativity and $n>1=$ positive cooperativity).

Substrate inhibition.

$$
v=\frac{\mathrm{V}_{\max }}{1+\frac{\mathrm{K}_{\mathrm{m}}}{[S]}+\frac{[S]}{\mathrm{K}_{\mathrm{si}}}}
$$

where $\mathrm{K}_{\mathrm{si}}$ is the substrate inhibition constant.

Intrinsic clearance $\left(\mathrm{Cl}_{\text {int }}\right)$ was calculated as $\mathrm{V}_{\max } / \mathrm{K}_{\mathrm{m}}$.

\section{Results}

\section{Opioid Glucosidation}

We have reported previously that both c-SUP and Supersomes expressing UGT2B7 catalyze the 3-glucosidation, but not the 6-glucosidation, of MOR in the presence of UDP-Glc as cofactor (Chau et al., 2014). Thus, the kinetics of MOR 3-glucosidation by c-SUP and Sf9 membranes were characterized using 11 substrate concentrations from 0.05 to $10 \mathrm{mM}$. Kinetic parameters are given as the mean of duplicate measurements \pm S.E. of the parameter fit. MOR 3-glucosidation by c-SUP exhibited hyperbolic (Michaelis-Menten) kinetics, whereas negative cooperative kinetics $(n=0.96 \pm 0.01)$ was observed with Sf9 membranes (Fig. 1). Mean $\mathrm{K}_{\mathrm{m}}\left(\right.$ or $\mathrm{S}_{50}$ ) values for MOR 3-glucosidation by c-SUP and Sf9 membranes were $3.4 \pm 0.001$ and $4.4 \pm 0.07 \mathrm{mM}$, respectively, and mean $\mathrm{V}_{\max }$ values were $266 \pm$ 9.3 and $362 \pm 2.5 \mathrm{pmol} / \mathrm{min} \cdot \mathrm{mg}$, respectively. In contrast to MOR, which has both phenolic (3-position) and enolic (6-position) hydroxyl groups, COD has only an enolic hydroxyl group at the 6-position. Consistent with the lack of MOR 6-glucosidation by c-SUP and Sf9 membranes, COD was not glucosidated by these enzyme sources.

\section{Activity of Uninfected Sf9 Membranes and c-SUP toward Hydroxyl-, Carboxylic Acid- and Amine-Containing Aglycones}

Screening studies were performed to further characterize the glucosidation capacity and selectivity of Sf9 membranes and c-SUP. Twelve substrates that contained either an aliphatic or aromatic (phenolic) phenolic hydroxyl group, or a carboxylic acid or amine functional group were investigated. The activity profile of each substrate was determined at four concentrations that provided a meaningful activity range while maintaining aglycone solubility in the incubation medium. 

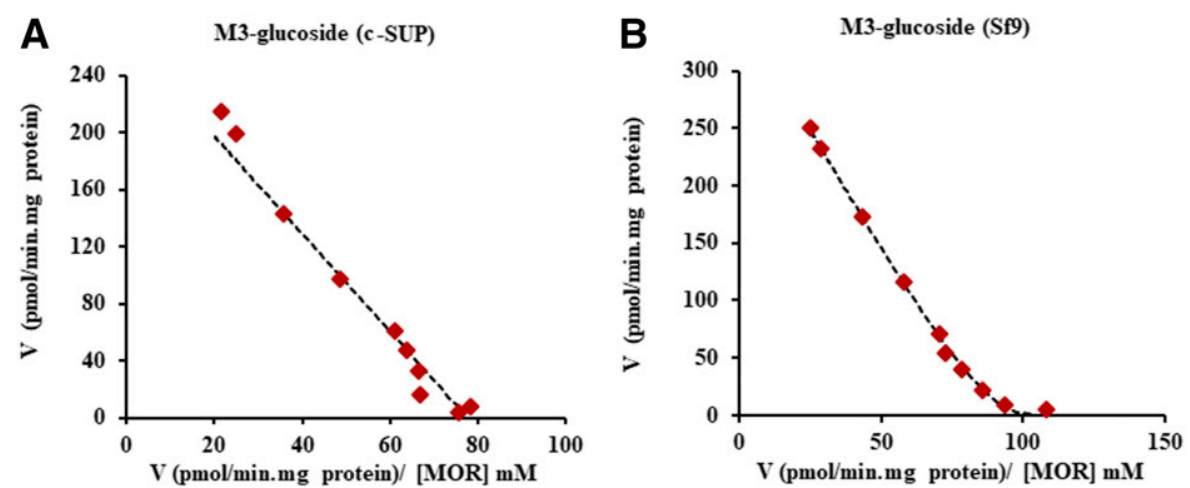

Fig. 1. Eadie-Hofstee plots for morphine 3-glucosidation by c-SUP (A) and uninfected $\mathrm{Sf} 9$ membranes (B). The substrate concentration range was $0.05-10 \mathrm{mM}$.

Glucosidation of Substrates Containing a Hydroxyl Group. In addition to MOR and COD, an additional eight compounds containing a phenolic or aliphatic hydroxyl group were screened for glucosidation by c-SUP and Sf9 membranes with UDP-Glc as cofactor (Fig. 2): 21-OHPr, 1-OHP, 4-MU, MPA, 1-NAP, 4-NP, PE, and AZT. AZT was glucosidated only by c-SUP (Fig. 2H). The rates of glucosidation of 21-OHPr, 1-OHP, and 1-NAP were higher with c-SUP compared with Sf9 membranes (Fig. 2, A, B, and E). At the highest aglycone concentration investigated, rates of glucosidation were approximately 22-, 28-, and 2.7-fold higher for 21-OHPr, 1-OHP, and 1-NAP, respectively. By contrast, the rates of formation of the glucosides of PE and MPA (phenolic) by Sf9 membranes were approximately 4- to 10.5-fold higher and 3- to 6-fold higher, respectively, compared with c-SUP (Fig. 2, D and H). The rates of 4-MU and 4-NP glucosidation were reasonably similar with both enzyme sources (Fig. 2, C and F).
To further characterize the glucosidation of hydroxyl-containing substrates, the kinetics of 1-OHP (Fig. 3, A and B), MPA (Fig. 3, C and D), and 4-MU (Fig. 3, E and F), glucosidation by c-SUP and Sf9 membranes were investigated using 11 or 12 aglycone concentrations that spanned the $\mathrm{K}_{\mathrm{m}}\left(\right.$ or $\mathrm{S}_{50}$ ). Substrate concentration ranges are given in the Fig. 3 caption. Best-fit kinetic equations were consistent with the activity data shown in Fig. 2, and as observed with MOR, the equation of best fit differed between the two enzyme sources for 1-OHP and MPA. 1-OHP glucosidation by c-SUP exhibited negative cooperative kinetics $(n=0.89 \pm 0.01)$ but weak substrate inhibition $\left(\mathrm{K}_{\mathrm{si}}=13.3 \pm 1.9 \mu \mathrm{M}\right)$ with $\mathrm{Sf9}$ membranes. Mean $\mathrm{K}_{\mathrm{m}}$ or $\mathrm{S}_{50}$ and $\mathrm{V}_{\max }$ values for 1-OHP glucosidation by c-SUP and Sf9 membranes were $8.0 \pm 0.21$ and $1.4 \pm 0.11 \mu \mathrm{M}$ and 11,211 \pm 144 and $2713 \pm 132 \mathrm{pmol} / \mathrm{min} \cdot \mathrm{mg}$, respectively. MPA phenolic glucosidation by c-SUP and Sf9 membranes was best described by
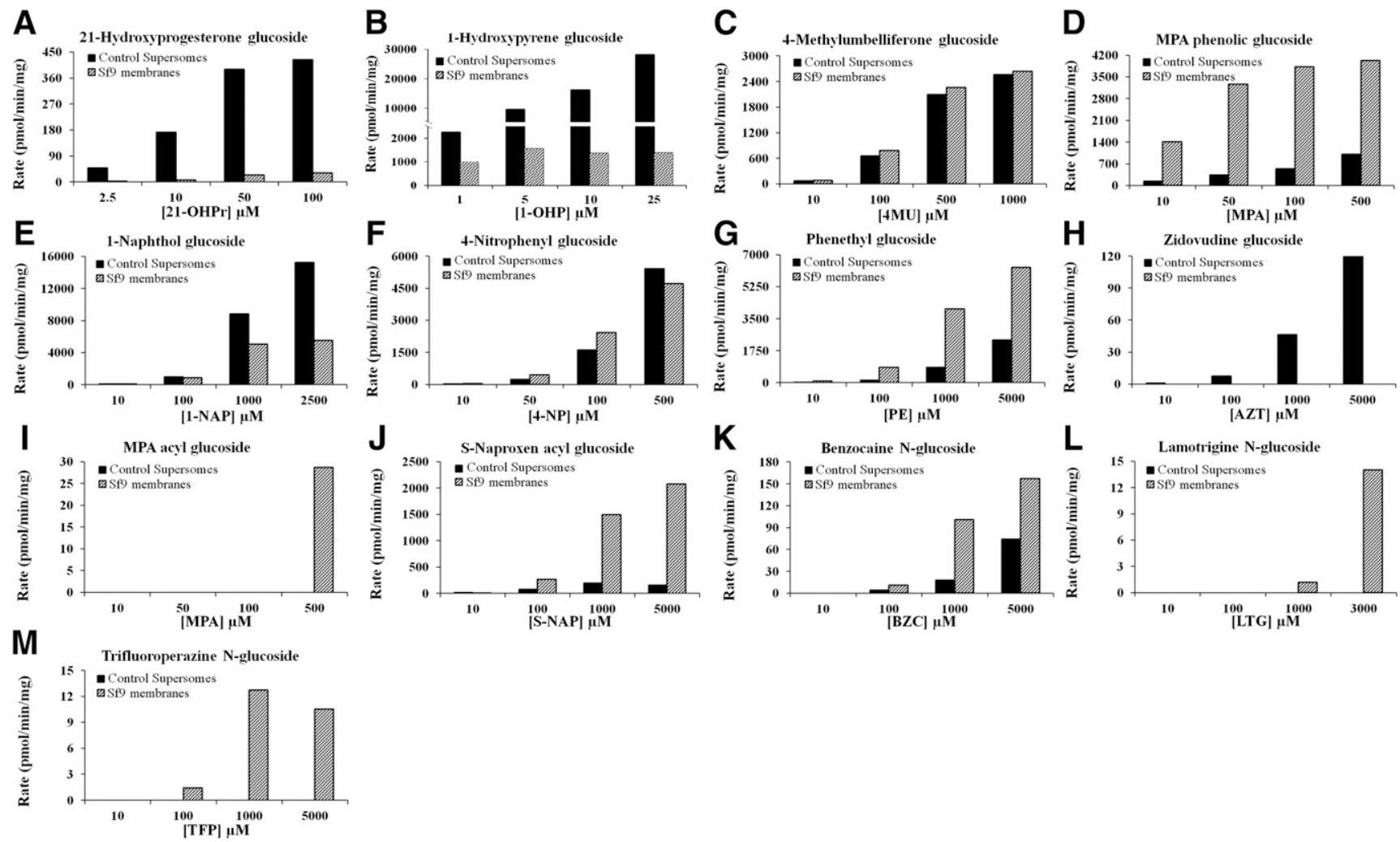

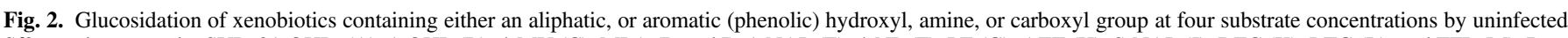

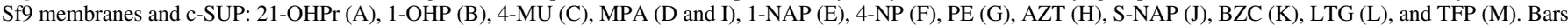
represent the mean of duplicate measurements $(<10 \%$ variance $)$. 
A

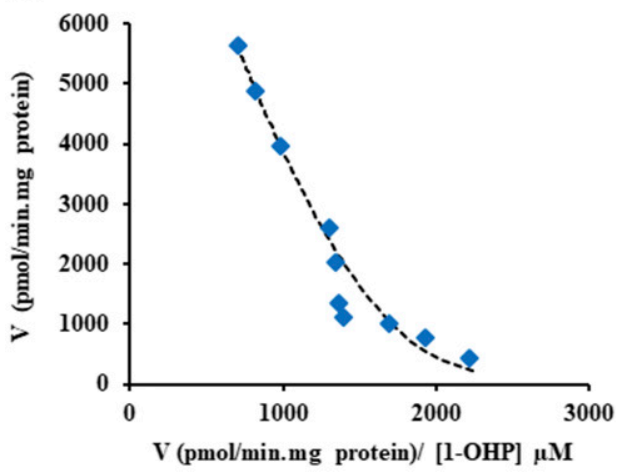

MPA phenolic glucoside (c-SUP)

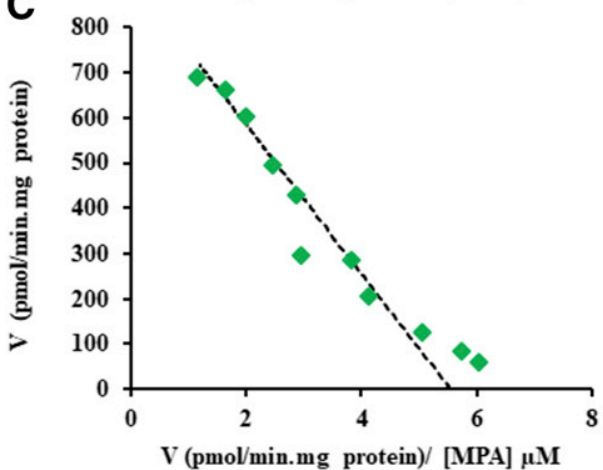

E

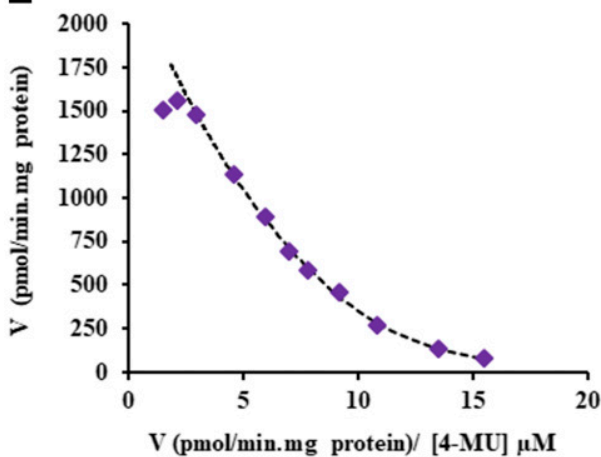

B
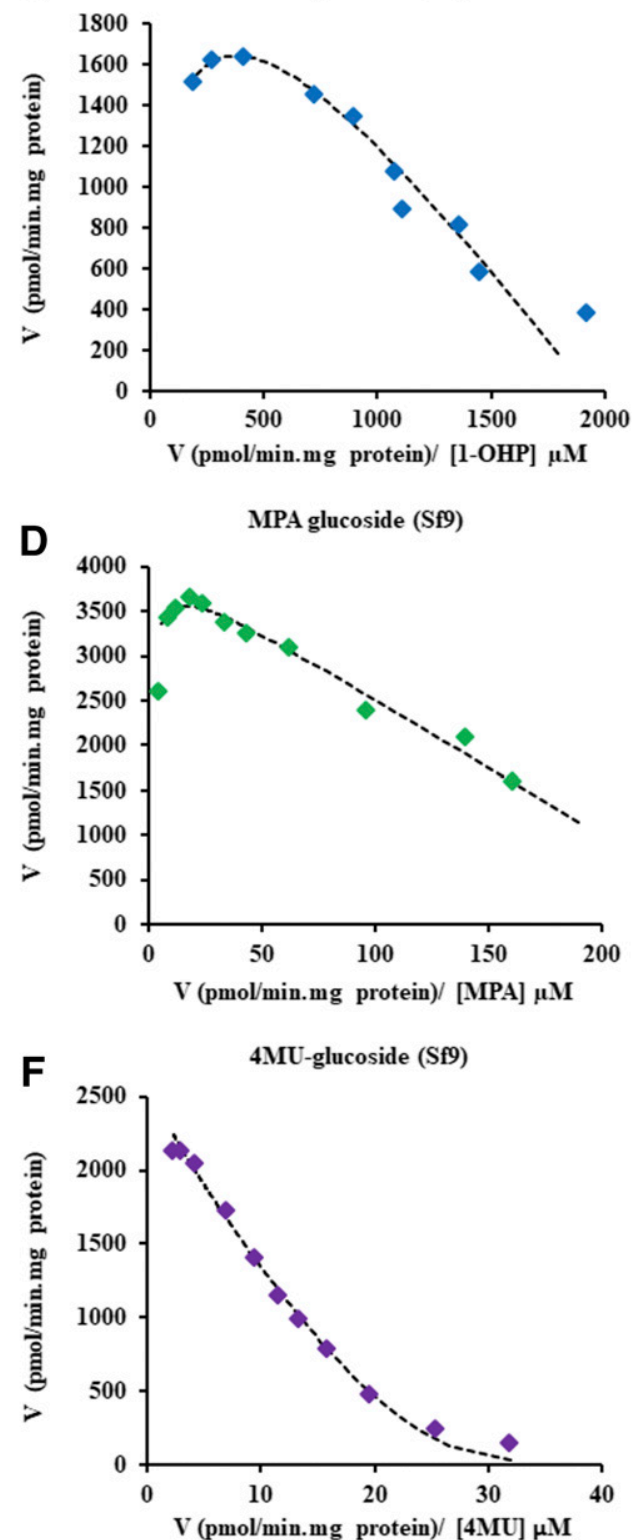

Fig. 3. Eadie-Hofstee plots for 1-OHP (A and $\mathrm{B})$, MPA (C and D), and 4-MU (E and F) glucosidation by c-SUP and uninfected $\mathrm{Sf} 9$ membranes. Substrate concentration ranges: 1-OHP, $0.2-8 \mu \mathrm{M}$; MPA, 10-600 $\mu \mathrm{M}$; and 4-MU, 5-1000 $\mu \mathrm{M}$. the Michaelis-Menten and substrate inhibition equations, respectively; mean $\mathrm{K}_{\mathrm{m}}$ and $\mathrm{V}_{\max }$ values for MPA phenolic glucosidation by c-SUP and Sf9 membranes were $165 \pm 0.35$ and $15.5 \pm 1.1 \mu \mathrm{M}$ $\left(\mathrm{K}_{\mathrm{si}}=2998 \pm 468 \mu \mathrm{M}\right)$, and $916 \pm 0.81$ and $4076 \pm 97 \mathrm{pmol} /$ min.mg, respectively. 4-MU glucosidation by both c-SUP and Sf9 membranes exhibited negative cooperative kinetics with mean $n$, $\mathrm{S}_{50}$, and $\mathrm{V}_{\max }$ values of $0.85 \pm 0.003,282 \pm 2.6 \mu \mathrm{M}$, and $2390 \pm$ $9.3 \mathrm{pmol} / \mathrm{min} \cdot \mathrm{mg}$, respectively, for c-SUP, and $0.91 \pm 0.03,123 \pm 8.3 \mu \mathrm{M}$, and $2580 \pm 63 \mathrm{pmol} / \mathrm{min} \cdot \mathrm{mg}$, respectively, for Sf9 membranes.

Glucosidation of Carboxylic Acid- and Amine-Containing Substrates. Rates of the acyl glucosidation of MPA and S-NAP, and the $N$-glucosidation of the amines BZC, LTG, and TFP by c-SUP and Sf9 membranes are shown in Fig. 2. Rates of S-NAP glucosidation were substantially higher (3- to 16-fold) with Sf9 membranes than with c-SUP (Fig. 2J). MPA acyl glucosidation was observed only with Sf9 membranes at the highest aglycone concentration (Fig. 2I). Similarly, LTG and TFP were glucosidated solely by Sf9 membranes (Fig. 2, L and
$\mathrm{M})$, and rates of BZC $\mathrm{N}$-glucosidation by $\mathrm{Sf9}$ membranes were more than double those of c-SUP (Fig. 2K).

\section{Verification of Glucoside Formation by c-SUP and Uninfected Sf9 Membranes}

Peaks corresponding to glucoside conjugates were not observed in chromatograms from experiments performed in the absence of UDP-Glc. As noted in Methods, authentic glucoside conjugates were available for $\mathrm{BZC}, 21-\mathrm{OHPr}, \mathrm{MPA}, 4-\mathrm{MU}$, and 4-NP. Glucosidation of these compounds was confirmed by comparison of HPLC retention times with those of authentic standards. In addition, the formation of a glucoside conjugate of the substrates investigated here was confirmed by LC-MS. Observed and predicted $\mathrm{m} / \mathrm{z}$ values for glucosides, except those of 4-NP, are shown in Table 1. In addition, fragmentation patterns were consistent with glucoside formation (data not shown). An $\mathrm{m} / \mathrm{z}$ value corresponding to 4-NP glucoside could not be detected by MS in positive ion mode, 
TABLE 1

Observed and predicted $\mathrm{m} / \mathrm{z}$ values (Daltons) of xenobiotic glucosides formed by incubations of uninfected Sf9 membranes and c-SUP with UDP-glucose as cofactor

\begin{tabular}{lccc}
\hline & & \multicolumn{2}{c}{ Observed Glucoside } \\
\cline { 2 - 4 } & Penobiotic & Sf9 Membranes & c-SUP \\
& & $m / z$ & $m / z$ \\
Benzocaine & $m / z$ & 328.13 & 328.13 \\
Codeine & 328.13 & ND & ND \\
21-Hydroxyprogesterone & 462.21 & 493.28 & 493.27 \\
1-Hydroxypyrene & 493.27 & 381.14 & 381.13 \\
Lamotrigine & 381.13 & ND \\
4-Methylumbelliferone & 418.07 & 418.07 & 339.09 \\
Morphine & 339.10 & 339.08 & 448.18 \\
Mycophenolic acid (phenolic and acyl) & 448.19 & 448.18 & 483.19 \\
1-Naphthol & 483.18 & 483.19 & 307.13 \\
S-Naproxen & 307.12 & 307.12 & $410.18^{a}$ \\
& $410.18^{a}$ & $410.16^{a}$ & $415.11^{b}$ \\
Phenethyl alcohol & $415.13^{b}$ & $415.13^{b}$ & $431.10^{c}$ \\
Trifluoperazine & $431.11^{c}$ & $431.11^{c}$ & 285.13 \\
Zidovudine & 285.13 & 285.15 & $\mathrm{ND}$ \\
\hline
\end{tabular}

$\mathrm{ND}$, not detected.

${ }^{a}$ S-naproxen $+\mathrm{NH}_{4}$ adduct.

${ }^{b} \mathrm{~S}$-naproxen $+\mathrm{Na}$ adduct.

${ }^{c} \mathrm{~S}$-naproxen $+\mathrm{K}$ adduct.

even for the authentic standard, despite detection by HPLC and UPLC. However, the fragmentation pattern was consistent with formation of 4-NP glucoside.

\section{Expression and Activity of UGT1A5 in Mammalian (HEK293T and COS7) and Insect (Sf9) Cell Lines}

Initial experiments sought to express human UGT1A5 in HEK293T and COS7 cells. Expression of UGT1A5 protein was not apparent in HEK293T cells (Fig. 4A), but expression was observed in COS7 cell lysate (Fig. 4B). Although 1-OHP has been reported to be glucuronidated by UGT1A5 (Finel et al., 2005), glucuronidation of this substrate was not observed with either the transfected HEK293T or COS7 cell lysates. 1-OHP glucuronidation was confirmed with human liver microsomes as the positive control (data not shown).

Given the lack of or relatively weak expression of UGT1A5 in the mammalian cell lines and the lack of observed 1-OHP glucuronidation activity, baculovirus-mediated expression of His-tagged UGT1A5 in Sf9 cells was undertaken. Western blot analysis using an antibody that recognizes His-tagged proteins identified a band with the expected molecular mass of UGT1A5 (Fig. 4C). As with the mammalian expression systems, the UGT1A5 protein expressed in Sf9 cells lacked glucuronidation activity toward 1-OHP. However, incubations of the enriched membrane fraction of Sf9 cells expressing UGT1A5 and uninfected Sf9 cells with UDP-GlcUA as the added cofactor showed the presence of a peak that chromatographed with almost the same retention time as 1-OHP glucuronide. The second peak was identified as 1-OHP glucoside by LC-MS and by comparison of the HPLC retention time with that of the authentic standard. Incubation of uninfected Sf9 cell membranes supplemented with UDP-Glc as cofactor resulted in the formation of a 1-OHP glucoside peak that had an approximate 800 -fold greater area than the peak formed with UDP-GlcUA as cofactor. The 1-OHP glucoside peak that formed with UDP-GlcUA as cofactor was presumed to arise from the presence of UDP-Glc as an impurity in commercial UDP-GlcUA. HEK293T, COS7, and Sf9 cells engineered to express recombinant UGT1A5 were additionally screened for 1-NAP, 4-MU, TFP, and LTG glucuronidation, but no activity was observed. UGT1A6 and UGT1A4 expressed in HEK293T cells were used as positive controls for the glucuronidation of 4-MU/1-NAP and TFP/LTG, respectively, as described by Uchaipichat et al. (2006) and Kubota et al. (2007).

\section{Discussion}

There is increasing evidence demonstrating that several human UGT1A and 2B subfamily enzymes may catalyze both glucuronidation and glucosidation reactions. Indeed, the importance of glucosidation as a drug and chemical biotransformation pathway may be underestimated (see Introduction). Recombinant UGT enzymes are used extensively for the reaction phenotyping of drug glucuronidation (Miners et al., 2010; Zientek and Youdim, 2015) and have also been used to investigate glucosidation (e.g., Mackenzie et al., 2003; Tang et al., 2003; Toide et al., 2004; Buchheit et al., 2011; Chau et al., 2014). Numerous mammalian and nonmammalian expression systems are used for the generation of recombinant UGT proteins, including baculovirusinfected insect (Sf9 and T. ni) cells (Radominska-Pandya et al., 2005). Recombinant human UGT enzymes expressed in insect cells are

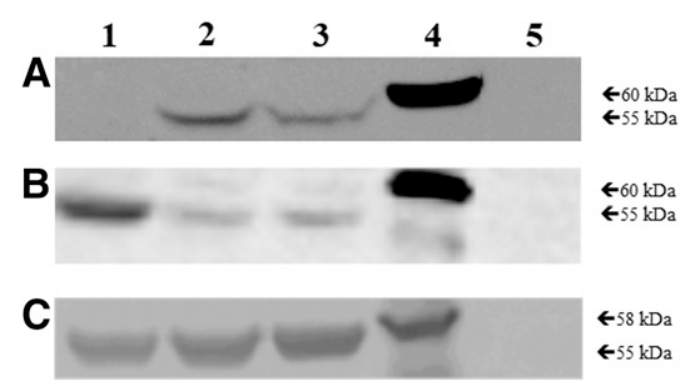

Fig. 4. Immunoblots of UGT1A5 expressed in HEK293T (Panel A), COS7 (Panel B) and Sf9 (Panel C) cells. Lane 1, wild-type UGT1A5; Lane 2, UGT1A5-His40Pro; Lane 3, UGT1A5-Thr36Ile; Lane 4, positive controls (UGT1A1 (panels A and B) and His-tagged CYP1A1 (panel C)); Lane 5, negative controls (untransfected HEK293T (panel A) and COS7 (panel B) cell lysate, and uninfected Sf9 (panel C) cell membranes). Immuno-reactive bands are observed at $55 \mathrm{kDa}$ for UGT1A5 and its mutants, $58 \mathrm{kDa}$ for CYP1A1 and $60 \mathrm{kDa}$ for UGT1A1. Western blots were performed in duplicate. 
available commercially (e.g., Baculosomes, Supersomes) and are used widely by both academic and industry laboratories. We reported previously that c-SUP efficiently catalyzed the glucosidation (with UDP-Glc as cofactor), but not glucuronidation, of MOR, suggesting that insect cells used for the generation of recombinant UGTs may express an endogenous UDP-glycosyltransferase(s) capable of glucosidating drugs and other chemicals. This prompted us to investigate the glucosidation of a series of aglycones with a phenolic (1-OHP, 4-MU, MOR, MPA, 1-NAP, and 4-NP), aliphatic alcohol (COD, 21-OHPr, PE, and AZT), acyl (MPA and S-NAP), or amine (BZC, LTG, and TFP) acceptor functional group (see Supplemental Fig. 1 for structures) by c-SUP and Sf9 membranes to characterize the scope and selectivity of drug and chemical glucosidation by these insect cell lines. All of the compounds investigated are known to be glucuronidated by human liver microsomes and/or recombinant UGTs (Green and Tephly, 1996; Shipkova et al., 2001; Stone et al., 2003; Uchaipichat et al., 2004, 2006; Finel et al., 2005; Rowland et al., 2006; Bowalgaha et al., 2007; Gaganis et al., 2007; Kubota et al., 2007; Raungrut et al., 2010).

Differences were observed in the substrate selectivities and activities of the native UDP-glucosyltransferases of c-SUP and Sf9 membranes. Among the phenols, rates of 1-OHP and 1-NAP glucosidation were substantially higher with c-SUP, while MPA was preferentially glucosidated by Sf9 membranes. Rates of glucosidation of 4-MU and 4-NP were similar with both enzyme sources. The aliphatic alcohols AZT and 21-OHPr were solely or preferentially glucosidated by c-SUP, while rates of PE glucosidation were higher with $\mathrm{Sf} 9$ membranes. Neither c-SUP nor Sf9 membranes glucosidated MOR and COD at the 6-position (enolic). Sf9 membranes glucosidated the carboxylic acid functional group of MPA and S-NAP and $N$-glucosidated BZC, LTG, and TFP. By contrast, glucosidation activity of c-SUP was not measurable (MPA, LTG, and TFP) or was low in comparison with Sf9 membranes (S-NAP and BZC).

Differences in the kinetics of 1-OHP, MPA, and MOR (3-position) glucosidation were also observed between the two enzyme sources: 1-OHP, negative cooperative (c-SUP) and substrate inhibition (Sf9); MPA, Michaelis-Menten (c-SUP) and substrate inhibition (Sf9); and MOR, Michaelis-Menten (c-SUP) and substrate inhibition (Sf9). By contrast, 4-MU glucosidation by both enzyme sources exhibited negative cooperative kinetics. When data are considered as intrinsic clearances (calculated as $\mathrm{K}_{\mathrm{m}}$ or $\mathrm{S}_{50}$ divided by $\mathrm{V}_{\max }$, noting that $n$ values were close to 1 for substrates exhibiting negative cooperative kinetics), ratios (c-SUP/Sf9 membranes) were of a similar order for 1-OHP (0.73), MOR (0.95), and 4-MU (0.40) but considerably lower for MPA (0.02). By way of comparison, the $\mathrm{K}_{\mathrm{m}} / \mathrm{S}_{50}$ values for MOR 3-glucosidation by c-SUP and Sf9 membranes $(3.42-4.40 \mathrm{mM})$ were similar to the $\mathrm{K}_{\mathrm{m}}$ (5.56 mM) reported for MOR 3-glucosidation by human liver microsomes, although the $\mathrm{V}_{\max }$ was lower (Chau et al., 2014). Notably, 1-OHP was glucosidated very efficiently by c-SUP and Sf9 membranes, with $\mathrm{Cl}_{\text {int }}$ values of 1409 and $1938 \mu \mathrm{l} / \mathrm{min} \cdot \mathrm{mg}$, respectively.

Taken together, the results demonstrate that c-SUP and Sf9 membranes have the capacity to glucosidate both drugs and nondrug xenobiotics. However, differences occur between the native UDPglucosyltransferases of c-SUP and Sf9 membranes. Although neither c-SUP nor Sf9 membranes catalyzed the 6-glucosidation of COD and MOR and $\mathrm{Cl}_{\text {int }}$ ratios were similar for several phenols (1-OHP, MOR, and 4-MU), Sf9 membranes preferentially glucosidated MPA while the aliphatic alcohols 21-OHPr and AZT were glucosidated almost exclusively by c-SUP. Sf9 membranes exclusively or preferentially glucosidated the carboxylic acid- and amine-containing aglycones investigated here.

While it is acknowledged that too few compounds were studied to establish meaningful structure-function relationships, it is apparent that care is required when investigating drug and chemical glucosidation by recombinant UGT enzymes expressed in insect cells. As noted previously, there is evidence demonstrating that UGT-catalyzed glucuronidation and glucosidation may occur as complementary metabolic pathways for xenobiotics. For example, we observed MOR 3-glucosidation by Supersomes expressing UGT2B4, UGT2B7, UGT2B15, and UGT2B17, but activity was only apparent for UGT2B7 when the background activity of c-SUP was taken into account (Chau et al., 2014). By contrast, HEK293 cells do not express an endogenous UDP-glycosyltransferase capable of glucosidating MOR and other xenobiotics (Chau et al., 2014). The data emphasize the requirement for "control" cell lysate/membranes in the investigation of drug and chemical glucosidation (and possibly conjugation with other sugars) by recombinant enzymes expressed in insect cells. It is known that many insect species, including lepidopterans (which include $S$. frugiperda and T. ni), express UDP-glycosyltransferases that preferentially use UDPGlc as cofactor for the metabolism of dietary and environmental chemicals (Ahn et al., 2012; Meech et al., 2012). It has also been proposed that insect viruses have evolved UDP-glycosyltransferases that apparently facilitate exploitation of insect larvae as hosts for reproduction (Meech et al., 2012), although it is unknown whether the viral vector (AcMNPV) used here expresses a xenobiotic UDP-glucosyltransferase.

Coincident with the study investigating xenobiotic glucosidation by c-SUP and uninfected Sf9 membranes, we commenced an investigation of UGT1A5 structure function. UGT1A5 expressed in Sf9 cells has been reported to readily glucuronidate 1-OHP (Finel et al., 2005). Using UDPGlcUA as cofactor, the rate of 1-OHP glucuronidation (at a substrate concentration of $500 \mu \mathrm{M}$ ) by UGT1A5 was $97 \mathrm{pmol} / \mathrm{min} \cdot \mathrm{mg}$. By contrast, rates of glucuronidation of 4-MU and scopoletin were low, approximately $1 \mathrm{pmol} / \mathrm{min} \cdot \mathrm{mg}$. In the present work, expression of UGT1A5 was observed in COS7 cells, but expression was not apparent in HEK293T cells using a commercial UGT1A subfamily antibody. Moreover, 1-OHP glucuronidation was not observed with lysates of COS7 and HEK293T cells, despite being readily measurable with human liver microsomes as the enzyme source (approximately $5000 \mathrm{pmol} /$ min.mg at a substrate concentration of $40 \mu \mathrm{M})$. Thus, we expressed Histagged UGT1 A5 in Sf9 cells as described by Finel et al. (2005), including use of the same cDNA. Despite demonstration of His-tagged UGT1A5 protein expression by immunoblotting, the enzyme did not glucuronidate 1-OHP and 4-MU. No product was observed that cochromatographed with authentic 1-OHP glucuronide or 4-MU glucuronide, nor was a peak with the expected $\mathrm{m} / \mathrm{z}$ ratio and fragmentation pattern for 1-OHP glucuronide observed using LC-MS. However, formation of 1-OHP glucoside was verified by HPLC and LC-MS. It was presumed that glucoside formation occurred due to the presence of UDP-Glc as an impurity in UDP-GlcUA, but this was not confirmed at the time. The stated purity of the UDP-GlcUA (trisodium salt) used in experiments is 98\%-100\%. There is anecdotal evidence suggesting that, at least in the past, UDP-Glc was present as an impurity in the trisodium salt of UDPGlcUA (but probably not in the triammonium salt). Use of UDP-Glc as cofactor with UGT1A5 expressed in Sf9 cells gave a 1-OHP glucoside peak with a peak area approximately 800 -fold higher that that observed with incubations conducted in the presence of UDP-GlcUA. In addition to wild-type UGT1A5, the Thr36Ile and His40Pro mutants were generated here. The mutants expressed in all three cell lines, albeit weakly in COS7 cells (Figure 4). Like wild-type UGT1A5, however, the mutants did not glucuronidate 1-OHP and 4-MU, nor the prototypic UGT1A4 substrates LTG and TFP (data not shown).

While artifactual glucosidation of 4-MU (and possibly scopoletin) may account for differences in the data presented here and by Finel et al. (2005), the relatively high rate of 1-OHP glucuronidation by UGT1A5 reported previously would seem inconsistent with glucosidation arising 
from the presence of UDP-Glc as an impurity in UDP-GlcUA. Nevertheless, identification of the glycoside conjugate(s) formed by incubations of insect cell membranes with UDP-GlcUA is recommended, especially when the rate of product formation is low. In addition to Finel et al. (2005) and the work presented here, UGT1A5 expressed in COS7 cells has been reported to glucuronidate 7-ethyl-10hydroxy-campothecin (SN-38), but the rate of glucuronidation was extremely low (ca. $100 \mathrm{pmol} / 16 \mathrm{~h} \cdot \mathrm{mg}$, equivalent to $0.1 \mathrm{pmol} / \mathrm{min} \cdot \mathrm{mg}$ ), and a nonspecific radiometric thin layer chromatographic method was used for product quantification (Ciotti et al., 1999). More recently, Yang et al. (2018) described the expression of active UGT1A5 and two polymorphic variants (UGT1A $5 * 8$ and UGT1A $5 * 9$ ) in fission yeast (Schizosaccharomyces pombe) cells. The activities of Triton X-100 permeabilized cells expressing the UGT1A5 enzymes were investigated using the UGT-Glo assay (Promega, Madison, WI), which measures the depletion of proluciferin substrates (UGT-Glo substrates A and B) rather than metabolite formation. Moreover, the activity (or lack thereof) of control (untransformed) fission yeast cells was not reported. Further studies are required to unambiguously characterize the functional role of UGT1A5.

\section{Acknowledgments}

Technical support from David Elliot is acknowledged. We are grateful to Dr. Andrew Rowland for his helpful advice.

\section{Authorship Contributions}

Participated in research design: Chau, Lewis, Mackenzie, Miners. Conducted experiments: Chau, Kaya.

Performed data analysis: Chau, Lewis, Miners.

Wrote or contributed to the writing of the paper: Chau, Lewis, Mackenzie, Miners.

\section{References}

Ahn SJ, Vogel H, and Heckel DG (2012) Comparative analysis of the UDP-glycosyltransferase multigene family in insects. Insect Biochem Mol Biol 42:133-147.

Bowalgaha K, Elliot DJ, Mackenzie PI, Knights KM, and Miners JO (2007) The glucuronidation of Delta4-3-Keto C19- and C21-hydroxysteroids by human liver microsomal and recombinant UDP-glucuronosyltransferases (UGTs): 6alpha- and 21-hydroxyprogesterone are selective substrates for UGT2B7. Drug Metab Dispos 35:363-370.

Buchheit D, Dragan CA, Schmitt EI, and Bureik M (2011) Production of ibuprofen acyl glucosides by human UGT2B7. Drug Metab Dispos 39:2174-2181.

Chau N, Elliot DJ, Lewis BC, Burns K, Johnston MR, Mackenzie PI, and Miners JO (2014) Morphine glucuronidation and glucosidation represent complementary metabolic pathways that are both catalyzed by UDP-glucuronosyltransferase 2B7: kinetic, inhibition, and molecular modeling studies. J Pharmacol Exp Ther 349:126-137.

Ciotti M, Basu N, Brangi M, and Owens IS (1999) Glucuronidation of 7-ethyl-10hydroxycamptothecin (SN-38) by the human UDP-glucuronosyltransferases encoded the UGT1 locus. Biochem Biophys Res Commun 260:199-202.

Fevery J, Van de Vijver M, Michiels R, and Heirwegh KPM (1977) Comparison in different species of biliary bilirubin-IX $\alpha$ conjugates with the activities of hepatic and renal bilirubin-IX $\alpha$-uridine diphosphate glycosyltransferases. Biochem J 164:737-746.

Finel M, Li X, Gardner-Stephen D, Bratton S, Mackenzie PI, and Radominska-Pandya A (2005) Human UDP-glucuronosyltransferase 1A5: identification, expression, and activity. J Pharmacol Exp Ther 315:1143-1149.

Fournel-Gigleux S, Sutherland L, Sabolovic N, Burchell B, and Siest G (1991) Stable expression of two human UDP-glucuronosyltransferase cDNAs in V79 cell cultures. Mol Pharmacol 39: $177-183$.

Gaganis P, Miners JO, Brennan JS, Thomas A, and Knights KM (2007) Human renal cortical and medullary UDP-glucuronosyltransferases (UGTs): immunohistochemical localization of UGT2B7 and UGT1A enzymes and kinetic characterization of S-naproxen glucuronidation. $J$ Pharmacol Exp Ther 323:422-430.

Green MD and Tephly TR (1996) Glucuronidation of amines and hydroxylated xenobiotics and endobiotics catalyzed by expressed human UGT1.4 protein. Drug Metab Dispos 24:356-363.

Jin C-J, Mackenzie PI, and Miners JO (1997) The regio- and stereo-selectivity of C19 and C21 hydroxysteroid glucuronidation by UGT2B7 and UGT2B11. Arch Biochem Biophys 341: 207-211.

Kerdpin O, Mackenzie PI, Bowalgaha K, Finel M, and Miners JO (2009) Influence of N-terminal domain histidine and proline residues on the substrate selectivities of human UDPglucuronosyltransferase 1A1, 1A6, 1A9, 2B7, and 2B10. Drug Metab Dispos 37:1948-1955.
Kubota T, Lewis BC, Elliot DJ, Mackenzie PI, and Miners JO (2007) Critical roles of residues 36 and 40 in the phenol and tertiary amine aglycone substrate selectivities of UDPglucuronosyltransferases 1A3 and 1A4. Mol Pharmacol 72:1054-1062.

Lu D, Dong D, Xie Q, Li Z, and Wu W (2018) Disposition of mianserin and cyclizine in UGT2B10-overexpressing human embryonic kidney 293 cells: identification of UGT2B10 as a novel $\mathrm{N}$-glucosidation enzyme and breast cancer resistance protein as an $\mathrm{N}$-glucoside transporter. Drug Metab Dispos 46:970-979.

Mackenzie PI, Bock KW, Burchell B, Guillemette C, Ikushiro S, Iyanagi T, Miners JO, Owens IS, and Nebert DW (2005) Nomenclature update for the mammalian UDP glycosyltransferase (UGT) gene superfamily. Pharmacogenet Genomics 15:677-685.

Mackenzie P, Little JM, and Radominska-Pandya A (2003) Glucosidation of hyodeoxycholic acid by UDP-glucuronosyltransferase 2B7. Biochem Pharmacol 65:417-421.

Mackenzie PI, Rogers A, Elliot DJ, Chau N, Hulin JA, and Miners JO, andMeech R (2011) The novel UDP glycosyltransferase 3A2: cloning, catalytic properties, and tissue distribution. Mol Pharmacol 79:472-478.

Mackenzie PI, Rogers A, Treloar J, Jorgensen BR, Miners JO, and Meech R (2008) Identification of UDP glycosyltransferase 3A1 as a UDP N-acetylglucosaminyltransferase. J Biol Chem 283: 36205-36210.

Meech R, Miners JO, Lewis BC, and Mackenzie PI (2012) The glycosidation of xenobiotics and endogenous compounds: versatility and redundancy in the UDP glycosyltransferase superfamily. Pharmacol Ther 134:200-218.

Miners JO, Mackenzie PI, and Knights KM (2010) The prediction of drug-glucuronidation parameters in humans: UDP-glucuronosyltransferase enzyme-selective substrate and inhibitor probes for reaction phenotyping and in vitro-in vivo extrapolation of drug clearance and drugdrug interaction potential. Drug Metab Rev 42:196-208.

Miners JO, Smith PA, Sorich MJ, McKinnon RA, and Mackenzie PI (2004) Predicting human drug glucuronidation parameters: application of in vitro and in silico modeling approaches. Annu Rev Pharmacol Toxicol 44:1-25.

Nguyen N and Tukey RH (1997) Baculovirus-directed expression of rabbit UDPglucuronosyltransferases in Spodoptera frugiperda cells. Drug Metab Dispos 25:745-749.

Ouzzine M, Magdalou J, Burchell B, and Fournel-Gigleux S (1999) An internal signal sequence mediates the targeting and retention of the human UDP-glucuronosyltransferase $1 \mathrm{~A} 6$ to the endoplasmic reticulum. $J$ Biol Chem 274:31401-31409.

Radominska-Pandya A, Bratton S, and Little JM (2005) A historical overview of the heterologous expression of mammalian UDP-glucuronosyltransferase isoforms over the past twenty years. Curr Drug Metab 6:141-160.

Raungrut P, Uchaipichat V, Elliot DJ, Janchawee B, Somogyi AA, and Miners JO (2010) In vitro-in vivo extrapolation predicts drug-drug interactions arising from inhibition of codeine glucuronidation by dextropropoxyphene, fluconazole, ketoconazole, and methadone in humans. J Pharmacol Exp Ther 334:609-618.

Rowland A, Elliot DJ, Williams JA, Mackenzie PI, Dickinson RG, and Miners JO (2006) In vitro characterization of lamotrigine N2-glucuronidation and the lamotrigine-valproic acid interaction. Drug Metab Dispos 34:1055-1062.

Senafi SB, Clarke DJ, and Burchell B (1994) Investigation of the substrate specificity of a cloned expressed human bilirubin UDP-glucuronosyltransferase: UDP-sugar specificity and involvement in steroid and xenobiotic glucuronidation. Biochem J 303:233-240.

Shipkova M, Strassburg CP, Braun F, Streit F, Gröne HJ, Armstrong VW, Tukey RH, Oellerich M, and Wieland E (2001) Glucuronide and glucoside conjugation of mycophenolic acid by human liver, kidney and intestinal microsomes. Br J Pharmacol 132:1027-1034.

Stone AN, Mackenzie PI, Galetin A, Houston JB, and Miners JO (2003) Isoform selectivity and kinetics of morphine 3- and 6-glucuronidation by human udp-glucuronosyltransferases: evidence for atypical glucuronidation kinetics by UGT2B7. Drug Metab Dispos 31:1086-1089.

Tang BK (1990) Drug glucosidation. Pharmacol Ther 46:53-56.

Tang C, Hochman JH, Ma B, Subramanian R, and Vyas KP (2003) Acyl glucuronidation and glucosidation of a new and selective endothelin ET(A) receptor antagonist in human liver microsomes. Drug Metab Dispos 31:37-45.

Toide K, Terauchi Y, Fujii T, Yamazaki H, and Kamataki T (2004) Uridine diphosphate sugarselective conjugation of an aldose reductase inhibitor (AS-3201) by UDPglucuronosyltransferase 2B subfamily in human liver microsomes. Biochem Pharmacol 67: 1269-1278.

Uchaipichat V, Mackenzie PI, Guo X-H, Gardner-Stephen D, Galetin A, Houston JB, and Miners JO (2004) Human udp-glucuronosyltransferases: isoform selectivity and kinetics of 4-methylumbelliferone and 1-naphthol glucuronidation, effects of organic solvents, and inhibition by diclofenac and probenecid. Drug Metab Dispos 32:413-423.

Uchaipichat V, Winner LK, Mackenzie PI, Elliot DJ, Williams JA, and Miners JO (2006) Quantitative prediction of in vivo inhibitory interactions involving glucuronidated drugs from in vitro data: the effect of fluconazole on zidovudine glucuronidation. Br J Clin Pharmacol 61:427-439. Yang F, Machalz D, Wang S, Li Z, Wolber G, and Bureik M (2018) A common polymorphic variant of UGT1A5 displays increased activity due to optimized cofactor binding. FEBS Lett 592:1837-1846.

Zhang H, Patana A-S, Mackenzie PI, Ikushiro S, Goldman A, and Finel M (2012) Human UDPglucuronosyltransferase expression in insect cells: ratio of active to inactive recombinant proteins and the effects of a C-terminal his-tag on glucuronidation kinetics. Drug Metab Dispos 40: $1935-1944$.

Zientek MA and Youdim K (2015) Reaction phenotyping: advances in the experimental strategies used to characterize the contribution of drug-metabolizing enzymes. Drug Metab Dispos 43: $163-181$.

Address correspondence to: John O. Miners, Department of Clinical Pharmacology, College of Medicine and Public Health, Flinders University, GPO Box 2100, Adelaide, SA 5001, Australia. E-mail: john.miners@flinders.edu.au 